\title{
Knowledge-Based Multiclass Support Vector Machines Applied to Vertical Two-Phase Flow
}

\author{
Olutayo O. Oladunni ${ }^{1}$, Theodore B. Trafalis ${ }^{1}$, and Dimitrios V. Papavassiliou ${ }^{2}$ \\ ${ }^{1}$ School of Industrial Engineering, The University of Oklahoma \\ 202 West Boyd, CEC 124 Norman, OK 73019 USA \\ \{tayo, ttrafalis\}@ou.edu \\ ${ }^{2}$ School of Chemical, Biological, and Materials Engineering, The University of Oklahoma \\ 100 East Boyd, SEC T-335 Norman, OK 73019 USA \\ dvpapava@ou.edu
}

\begin{abstract}
We present a knowledge-based linear multi-classification model for vertical two-phase flow regimes in pipes with the transition equations of McQuillan \& Whalley [1] used as prior knowledge. Using published experimental data for gas-liquid vertical two-phase flows, and expert domain knowledge of the two-phase flow regime transitions, the goal of the model is to identify the transition region between different flow regimes. The prior knowledge is in the form of polyhedral sets belonging to one or more classes. The resulting formulation leads to a Tikhonov regularization problem that can be solved using matrix or iterative methods.
\end{abstract}

\section{Introduction}

Multiphase flow in a pipe causes certain flow patterns to appear depending on pipe size, flow rates, fluid properties, and pipe inclination angle (when appropriate). Considerable progress has been made in defining such flow patterns [1-5], however there is no exact theory for their characterization. There is often disagreement about the transition boundaries between flow patterns making the selection of appropriate flow correlations for the description of the flow difficult. Therefore, it is important to develop a flow pattern model that minimizes the rate of misclassification errors (i.e., predicting the wrong flow regime for a given set of flow data) as well as extend the applicability of any new multiphase flow correlation to different pipe sizes, flow rates and fluid properties.

More recently, attempts have been made to identify multiphase flow regimes using non-linear classifiers derived from machine learning algorithms [6-8]. Trafalis et al. [9] employed a multi-classification support vector machine (MSVM) model, in which the superficial velocities and pipe sizes were used to detect flow regime transitions for two-phase flow in pipes. The model was data driven, and it outperformed the theoretical vertical flow correlation in terms of correct flow regime classification.

The primary objective of the present paper is to extend the model of [9] by exploring the effects of expert knowledge on the MSVM model. If one has prior information regarding a class or label, representing such knowledge and incorporating it into a classification problem can lead to a more robust solution. The vertical two-phase flow 
regimes considered herein are bubble flow (class 1), intermittent flow (slug \& churn flow, class 2), and annular flow (class 3). These flow regimes occur for a given set of parameters, (i.e., flow rates, fluid properties and pipe diameter). The theoretical correlations of McQuillan and Whalley [1], which were found to perform well for vertical two phase flow in [9], are used here as prior knowledge.

\section{Linear Multiclassification Tikhonov Regularization Knowledge-Based Support Vector Machine: Pairwise Knowledge Set Formulation}

Recently, prior knowledge formulation, incorporation, and solution have been studied in both the context of function approximations [10] and in the context of classifiers [11]. Here, we consider a problem of classifying data sets in $R^{n}$ that are represented by a data matrix $A^{i} \in R^{m_{i} \times n}$, where $i=1, . ., k$ ( $k \geq 2$ classes). Let $A^{i}$ be an $m_{i} \times n$ matrix whose rows are points in the $i^{\text {th }}$ class, and $m_{i}$ is the number of data in class $i$. Let $A^{j}$ be an $m_{j} \times n$ matrix whose rows are points in the $j^{\text {th }}$ class, and $m_{j}$ is the number of data in class $j$, and $y^{i j}= \pm 1$ for classes $i$ and $j$, respectively. This problem can be modeled through the following optimization problem:

$$
\begin{aligned}
& \min _{w, \gamma, \xi} \frac{\lambda}{2} \sum_{i<j}^{k}\left\|w^{i j}\right\|^{2}+\frac{1}{2} \sum_{i<j}^{k} \sum_{t=1}^{m_{i j}}\left(\xi_{t}^{i j}\right)^{2} \\
& \text { s.t. } \xi_{,}^{i j}=y_{t}^{i j}\left(A_{t}^{i j} w^{i j}-\gamma^{i j}\right)-1, \quad t=1, \ldots ., m_{i j} \\
& \text { where } m_{i j}=m_{i}+m_{j}, A^{i j}=\left(\begin{array}{c}
A^{i} \\
A^{j}
\end{array}\right), \quad y^{i j}=\left(\begin{array}{c}
y^{i} \\
y^{j}
\end{array}\right)=\left(\begin{array}{c}
+1 \\
-1
\end{array}\right)
\end{aligned}
$$

Above, $\lambda$ is the tradeoff constant, and $\xi^{i j}$ are error slacks measuring the deviation of points from their respective bounding planes. Classification weights $\left(w^{i j}, \gamma^{i j}\right)$ characterize the optimal separating planes

$$
x^{T} w^{i j}=\gamma^{i j}, \quad i<j .
$$

The locations of the optimal separating planes relative to the origin are determined by the value of $\gamma^{i j}$. Problem (2.1) is minimized parametrically with the tradeoff constant $\lambda$, which accounts for the tradeoff between minimum norm and minimum misclassification error.

Now, suppose that in addition to the classification problem, there is prior information belonging to one or more categories. The knowledge sets in $n$ dimensional space are given in the form of a polyhedral set determined by the set of linear equalities and linear inequalities. The given knowledge sets are (see Fig. 1)

$$
\left\{x \mid B^{i} x \leq b^{i}\right\} \text { or }\left\{x \mid \bar{B}^{i} x=\bar{b}^{i}\right\} \text {, belonging to class } i \text {, and }
$$

$g_{i}$ or $\bar{g}_{i}$ are the number of prior knowledge (equality or inequality) constraints in class $i$. 


$$
\left\{x \mid B^{j} x \leq b^{j}\right\} \text { or }\left\{x \mid \bar{B}^{j} x=\bar{b}^{j}\right\}, \text { belonging to class } j, \text { and }
$$

$d_{j}$ or $\bar{d}_{j}$ are the number of prior knowledge (equality or inequality) constraints in class $j$.

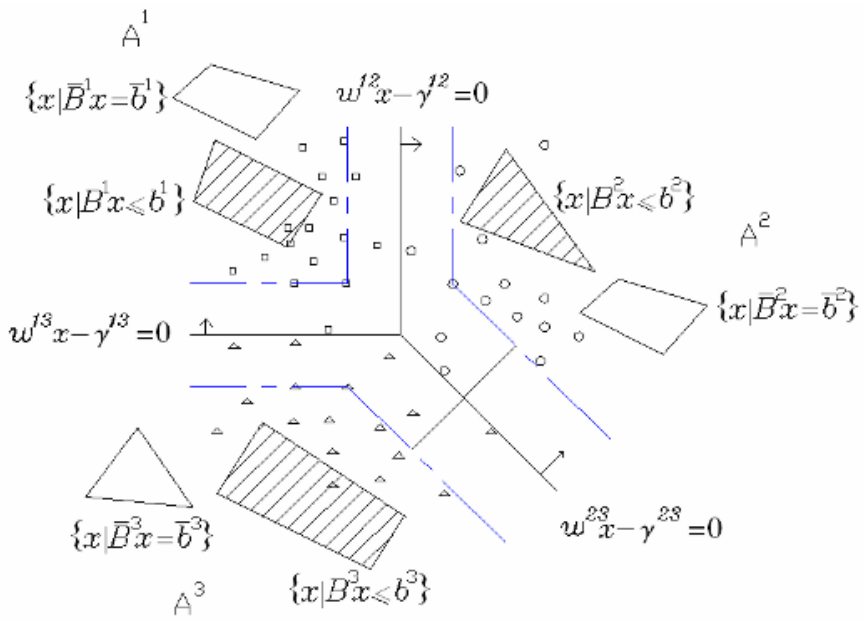

Fig. 1. A knowledge-based classification diagram

One can rewrite the constraints, and create an unconstrained optimization problem, called Linear Multi-classification Tikhonov Regularization Knowledge-Based Support Vector Machine $\left(\mathrm{L}_{\mathrm{M}} \mathrm{T}_{\mathrm{R}} \mathrm{KSVM}\right)[12,13,14]$.

$$
\min _{w, \gamma, \xi, u, v} f_{L_{M} T_{R} K S V M}(w, \gamma, \xi, u, v)=\left[\begin{array}{l}
\frac{\lambda}{2}\|w\|^{2}+\frac{1}{2}\|A w+E \gamma-e\|^{2}+ \\
\frac{1}{2}\left[\left\|B_{u}^{T} u+I_{u} w\right\|^{2}+\left\|B_{v}^{T} v-I_{v} w\right\|^{2}\right]+ \\
\frac{1}{2}\left[\left\|B_{b u}^{T} u+E_{u} \gamma+e_{u}\right\|^{2}+\left\|B_{b v}^{T} v-E_{v} \gamma+e_{v}\right\|^{2}\right]
\end{array}\right] .
$$

Where $u=\left[u^{i j T}, \ldots, u^{(k-1) k T}, \bar{u}^{i j T}, \ldots ., \bar{u}^{(k-1) k T}\right]^{T}$ is the vector of all multipliers referring to the $i^{\text {th }}$ class, and $v=\left[v^{i j T}, \ldots, v^{(k-1) k T}, \bar{v}^{i j T}, \ldots ., \bar{v}^{(k-1) k T}\right]^{T}$ is the vector of all multipliers referring to the $j^{\text {th }}$ class. $\xi$ is a residual error vector accounting for the training data error, and vectors $r, s, p, \sigma$ are residual error vectors accounting for the knowledge set error (i.e., violation of the knowledge constraints). The following matrices are defined for all $i<j, \quad i, j=1, \ldots . k$ classes (note that matrices describe the formation of the prior knowledge): Matrices $A^{i} \in R^{m_{i} \times n}, A^{j} \in R^{m_{j} \times n}, i<j$ are matrices whose rows belong to the $i^{t h}$ and $j^{\text {th }}$ class respectively; $e^{i}, e^{j} \in R^{m_{i}, m_{j} \times 1}$ are vectors of ones. Matrices 
$B_{u}$ and $B_{v}$ are diagonal block matrices whose diagonals contain knowledge sets belonging to the $i^{\text {th }}$ (or $j^{\text {th }}$ ) class. The $i^{\text {th }}$ (or $j^{\text {th }}$ ) knowledge set is derived from the inequality and equality prior knowledge constraints. The diagonals of $B_{u}, B_{v}$ are $B_{i j}^{i}, \bar{B}_{i j}^{i} \in R^{g_{i} \times n}\left(B_{i j}^{j}, \bar{B}_{i j}^{j} \in R^{g_{j} \times n}\right)$. Matrices $B_{b u}, B_{b v}$ are matrices consisting of vectors $b_{i j}^{i}, \bar{b}_{i j}^{i} \in R^{g_{1} \times 1}\left(b_{i j}^{j}, \bar{b}_{i j}^{j} \in R^{g_{1} \times 1}\right)$ and each component of these vectors describes the bounds of the knowledge set. Matrices $I_{u}, I_{v}$ are block matrices consisting of identity matrices $I_{u i j}, \bar{I}_{u j j} \in R^{n \times n}\left(I_{v i j}, \bar{I}_{v i j} \in R^{n \times n}\right)$. Matrices $E_{u}, E_{v}$ are matrices consisting of entries $e_{u i j}, \bar{e}_{u i j} \in R\left(e_{v i j}, \bar{e}_{v i j} \in R\right)$, where $e_{u i j}, \bar{e}_{u i j}$ is equal to one. Vector $e_{u}\left(e_{v}\right)$ is a vector of ones, where each entry corresponds to a vector $b_{i j}^{i}, \bar{b}_{i j}^{i} \in R^{g_{1} \times 1}\left(b_{i j}^{j}, \bar{b}_{i j}{ }^{j} \in R^{g_{j} \times 1}\right)$. Each $i^{\text {th }}$ and $j^{\text {th }}$ entry into $B_{u}, B_{b u}, I_{u}, E_{u}, e_{u}$, and $B_{v}, B_{b v}, I_{v}, E_{v}, e_{v}$ completes a pairwise comparison. The formulation is applicable to $k \geq 2$ [14].

Problem (2.5) is the unconstrained $\mathrm{L}_{\mathrm{M}} \mathrm{T}_{\mathrm{R}} \mathrm{KSVM}$ model which incorporates the knowledge sets (2.3) and (2.4) into the $\mathrm{L}_{M} \mathrm{~T}_{\mathrm{R}} \mathrm{SVM}$ model (2.1). The tradeoff constant $\lambda$ is also run through a range of values to achieve the best result. If its value increases then the minimum norm is achieved, but at the expense of having a higher training residual error and higher knowledge set residual error. If data is not available, the second term of the $\mathrm{L}_{\mathrm{M}} \mathrm{T}_{\mathrm{R}} \mathrm{KSVM}$ model can be dropped. This result in classifiers based strictly on knowledge sets, and it is useful for situations in which only expert knowledge exists. The $\mathrm{L}_{2}$ norm of all terms is considered because of its strong convexity of the objective function. Problem (2.5) is a convex unconstrained optimization problem that has a unique minimum point [14]. The decision function for classifying a point is given by:

$$
D(x)=\operatorname{sign}\left[x^{T} w^{i j}-\gamma^{i j}\right]=\left\{\begin{array}{l}
+1, \text { if point }(x) \text { is in class } A^{i} \\
-1, \text { if point }(x) \text { is in class } A^{j}
\end{array} .\right.
$$

A new point $x$ is classified according to the voting approach $[15,16]$. For example, if sign $\left[x^{T} w^{i j}-\gamma^{i j}\right]$ indicates that $x$ belongs to class $i$, then the vote for class $i$ is increased by one (otherwise $j$ is increased by one). Finally, $x$ belongs to the class with the largest vote. If two classes have identical votes, then select the one with the smallest index.

\section{Vertical Two-Phase Flow Data and Prior Knowledge}

Fifty percent of each data set described below were trained on the $\mathrm{L}_{\mathrm{M}} \mathrm{T}_{\mathrm{R}} \mathrm{KSVM}$ model, and the other $50 \%$ were used as test samples (for data source details see [9]).

2D classification (data): The 2D vertical flow data set uses two flow rates (superficial gas and liquid velocity) for one inch diameter pipes, with fluid properties at atmospheric conditions, to delineate three different flow regimes. There are 209 instances (points) and 2 attributes (features), 107 points were used as training samples and 102 
points were used as test samples. The distribution of instances with respect to their class is as follows: 44 instances in class 1 (bubble flow), 102 instances in class 2 (intermittent flow), and 63 instances in class 3 (annular flow).

3D classification (data): The 3D vertical flow data set uses the pipe size in addition to the superficial gas and liquid velocity to delineate three different flow regimes. There are 424 instances, and 3 attributes, 206 data points used as training samples, 218 points used as test samples. The distribution of instances is 98 instances in class 1 (bubble flow), 217 instances in class 2 (intermittent flow), and 109 instances in class 3 (annular flow).

Prior Knowledge: In addition to the vertical flow data, prior knowledge is included to develop knowledge based classification models. Since the flow regime data are scaled by taking the natural logarithm of each instance, the prior knowledge also needs to be scaled. Below are the transition equations $[1,9]$ and their equivalent logarithmic transformations for 2D and 3D knowledge based classification:

- Bubble - intermittent flow transition

$$
\begin{aligned}
& v_{L S}=3.0 v_{G S}-1.15\left[\frac{g \sigma\left(\rho_{L}-\rho_{G}\right)}{\rho_{L}^{2}}\right]^{1 / 4} \\
& \Rightarrow-0.9883 \ln \left(v_{G S}\right)+\ln \left(v_{L S}\right)=1.0608, \text { For both 2D \& 3D classification }
\end{aligned}
$$

- Bubble - dispersed bubble flow transition

$$
\begin{aligned}
& v_{L S} \geq \frac{6.8}{\rho_{L}^{0.444}}\left\{g \sigma\left(\rho_{L}-\rho_{G}\right)\right\}^{0.278}\left(\frac{D}{\mu_{L}}\right)^{0.112} \\
& \Rightarrow\left\{\begin{array}{l}
\ln \left(v_{L S}\right) \geq 1.03345,2 \mathrm{D} \text { classification } \\
\ln \left(v_{L S}\right) \geq 1.4466+0.112 \ln (D), 3 \mathrm{D} \text { classification }
\end{array}\right.
\end{aligned}
$$

Above, $D$ is the pipe diameter, $\mathrm{g}$ is the acceleration due to gravity, $v_{G S}, v_{L S}$ are the gas and liquid superficial velocities, respectively, $\rho_{G}, \rho_{L}$ are the gas and liquid densities, $\sigma$ is the surface tension, and $\mu_{G}, \mu_{L}$ are the gas and liquid viscosities, respectively. The correlations were selected based on the uncertainty of the transition lines as evidenced by the misclassification errors of the MSVM [9]. Also, as a result of the uncertainty, the threshold values of the vertical and horizontal correlations were deviated by a small deviation factor, $\pm \varepsilon$. This is in fact necessary because the correlation equations identify points on transition boundaries that have no distinct flow regime. So a small deviation of the thresholds facilitates the formation of bounds (deviated thresholds) for each flow regime. For instance equation (3.1), 2D case will be represented by

$$
\begin{aligned}
& -0.9883 \ln \left(v_{G S}\right)+\ln \left(v_{L S}\right) \geq 1.0608(1+\varepsilon) \rightarrow \text { Bubble } . \\
& -0.9883 \ln \left(v_{G S}\right)+\ln \left(v_{L S}\right) \leq 1.0608(1-\varepsilon) \rightarrow \text { Intermitent }
\end{aligned}
$$




\section{Computational Results}

In this section, the results of the analyzed data sets and prior sets described in section 3 are presented and discussed. The $\mathrm{L}_{M} \mathrm{~T}_{\mathrm{R}} \mathrm{KSVM}$ model is used to train the data sets with prior knowledge. To compare between the different models, a performance parameter (misclassification error) was defined as the fraction of misclassified points for a given data set

$$
\beta=1-\left(\frac{\text { Total number of correctly classified points }}{\text { Total number of observed points }}\right) .
$$

The tradeoff constants considered are between $0-100$, and the deviation factors considered are within the interval $0.01-0.1$. The outputs (flow regimes) were coded as: 1 - bubble, 2 - intermittent (slug and churn) and 3 - annular.

Results of the 2D \& 3D vertical flow data with prior knowledge information trained on the $\mathrm{L}_{\mathrm{M}} \mathrm{T}_{\mathrm{R}} \mathrm{KSVM}$ model and compared with the $\mathrm{L}_{\mathrm{M}} \mathrm{T}_{\mathrm{R}} \mathrm{SVM}$ model (2.1), are shown in Fig. $2 \& 3$. It should be noted that CPU time is measured in seconds. The theoretical correlations of McQuillan \& Whalley [1] were also simulated to compare with the learning models. The error rate is 0.0163 for the $2 \mathrm{D}$ vertical flow data and 0.1227 for the $3 \mathrm{D}$ vertical flow data. All computations were performed using MATLAB [18]. In bold face are the lowest error rates for each tradeoff constant $\lambda$.

Fig. 2 presents the average test error rate results, based on three random samples for the 2D vertical flow data with prior knowledge as defined in section 4 . The $\mathrm{L}_{\mathrm{M}} \mathrm{T}_{\mathrm{R}} \mathrm{SVM}$ and $\mathrm{L}_{\mathrm{M}} \mathrm{T}_{\mathrm{R}} \mathrm{KSVM}$ models generally report promising error rates. The error rate $(0.0098)$ for the model with prior knowledge $\left(\mathrm{L}_{M} \mathrm{~T}_{\mathrm{R}} \mathrm{KSVM}\right)$ is the same as the one for the data driven model $\left(\mathrm{L}_{\mathrm{M}} \mathrm{T}_{\mathrm{R}} \mathrm{SVM}\right)$, but smaller than the error rate of the theoretical correlations for $2 \mathrm{D}$ vertical flow. Fig. 3 presents the average test error rate results, based on four runs with random samples for the $3 \mathrm{D}$ vertical flow data with prior knowledge. The model with prior knowledge (error rate of 0.0413 ) performs better than both the data driven model and the 3D vertical flow theoretical model.

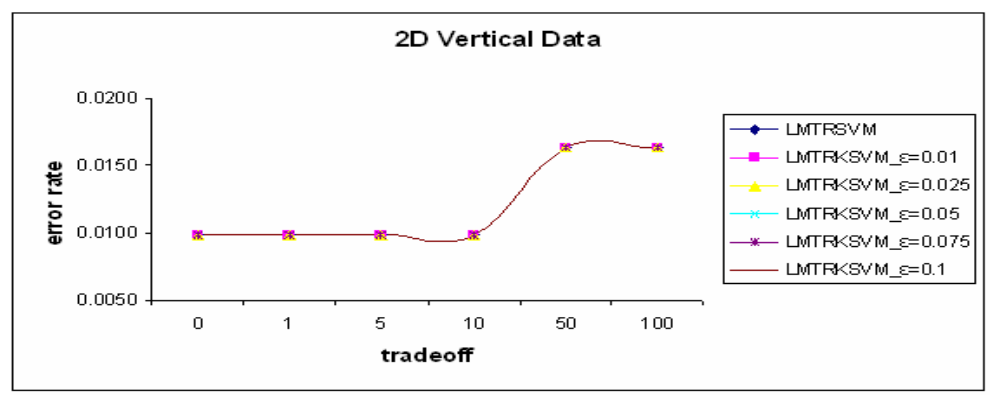

Fig. 2. Average test error rate for $L_{M} T_{R} K S V M$ on $2 D$ vertical flow data (varying tradeoff) 


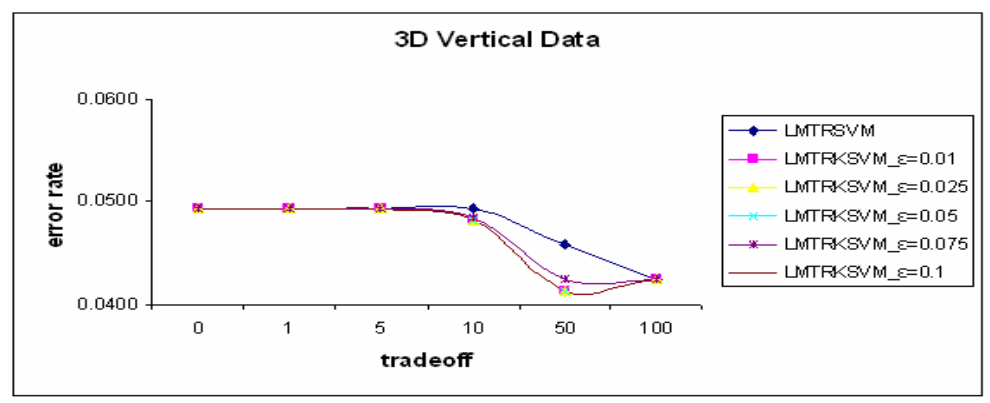

Fig. 3. Average test error rate for $L_{M} T_{R} K S V M$ on $3 D$ vertical flow data (varying tradeoff)

Irrespective of the deviation factor, $\varepsilon$, the prior knowledge models appear to perform better or at least display equal level of performance (error rate) with the data driven model $\left(\mathrm{L}_{M} \mathrm{~T}_{\mathrm{R}} \mathrm{SVM}\right)$. For all data sets, the $\mathrm{L}_{M} \mathrm{~T}_{R} \mathrm{KSVM}$ performs better than the theoretical model. However, since most of the theoretical correlations are nonlinear models, a nonlinear classification of the data is likely to achieve better generalization ability and lower error rate (note that the present model assumes that the data are linearly separable).

\section{Conclusion}

In this paper, a knowledge-based multi-classification model called the Linear Multiclassification Tikhonov Regularization Knowledge-based Support Vector Machine $\left(\mathrm{L}_{\mathrm{M}} \mathrm{T}_{\mathrm{R}} \mathrm{KSVM}\right.$ ) was applied to vertical, two-phase flow data and comparisons were made with a data driven model $\left(\mathrm{L}_{\mathrm{M}} \mathrm{T}_{\mathrm{R}} \mathrm{SVM}\right)$ and a theoretical model. The $\mathrm{L}_{\mathrm{M}} \mathrm{T}_{\mathrm{R}} \mathrm{KSVM}$ model using pipe size and superficial velocities as input training vector attributes of the flow regime produces a better overall misclassification error in comparison to theoretical models. Increasing the dimensionality of the classification problem to $3 \mathrm{D}$ for the vertical flow data still produces better error rates for $\mathrm{L}_{\mathrm{M}} \mathrm{T}_{\mathrm{R}} \mathrm{KSVM}$ models. The theoretical model error rate is quite large and clearly out of the error rate interval of the $\mathrm{L}_{M} \mathrm{~T}_{\mathrm{R}} \mathrm{KSVM} 3 \mathrm{D}$ vertical flow model. This highlights the strengths of the prior knowledge learning model.

\section{References}

1. McQuillan, K.W., Whalley, P.B.: Flow Patterns in Vertical Two-Phase Flow. Int. J. Multiphase Flow, (1985) 11, 161 - 175

2. Mandhane, J.M., Gregory, G.A., Aziz, K.: A Flow Pattern Map for Gas-Liquid Flow in Horizontal Pipes. Int. J. Multiphase Flow, (1974) 1, 537 - 553

3. Taitel, Y., Bornea, D., Dukler, A.E.: Modeling Flow Pattern Transitions for Steady Upward Gas-Liquid Flow in Vertical Tubes. AIChE J., (1980) 26, 345

4. Taitel, Y., Dukler, A.E.: A Model for Predicting Flow Regime Transitions in Horizontal and Near Horizontal Gas-Liquid Flow. AIChE J, (1976) 22, 47 
5. Petalas, N., Aziz, K.: A Mechanistic Model for Multiphase Flow in Pipes. CIM98-39, Proceedings, 49th Annual Technical Meeting of the Petroleum Society of the CIM, Calgary, Alberta, Canada, (1998) June 8-10

6. Osman, E.A.: Artificial Neural Networks Models for Identifying Flow Regimes and Predicting Liquid Holdup in Horizontal Multiphase Flow. SPE 68219, (2000) March

7. Mi, Y., Ishii, M., Tsoukalas, L.H.: Flow Regime Identification Methodology with Neural Networks and Two-Phase Flow Models. Nuclear Engineering and Design, (2001), 204, 87 - 100

8. Ternyik, J., Bilgesu, H.I., Mohaghegh, S.: Virtual Measurement in Pipes, Part 2: Liquid Holdup and Flow Pattern Correlations. SPE 30976, (1995) September

9. Trafalis, T.B., Oladunni, O., Papavassiliou, D.V.: Two-Phase Flow Regime Identification with a Multi-Classification SVM Model. Industrial \& Engineering Chemistry Research, (2005) 44, $4414-4426$

10. Mangasarian, O.L., Shavlik, J.W., Wild, E.W.: Knowledge-Based kernel approximation. Journal of Machine Learning Research, 5, 1127-1141, (2004)

11. Fung, G., Mangasarian, O.L., Shavlik, J.W.: Knowledge-Based support vector machine classifiers. Neural Information Processing Systems 2002 (NIPS 2002), Vancouver, BC, December 10-12, (2002). “Neural Information Processing Systems 15", S. Becker, S. Thrun and K. Obermayer, editors, MIT Press, Cambridge, MA, 2003, 521-528

12. Tikhonov, A.N., Arsenin, V.Y.: Solution of Ill-Posed Problems. Winston, Washington D.C., (1977)

13. Pelckmans, K., Suykens, J.A.K., De Moor, B.: Morozov, Ivanov and Tikhonov regularization based LS-SVMs. In Proceedings of the International Conference On Neural Information Processing (ICONIP 2004), Calcutta, India, Nov. 22-25, (2004)

14. Oladunni, O., Trafalis, T.B.: Linear Multi-classification Tikhonov Regularization Knowledge-based Support Vector Machine $\left(\mathrm{L}_{\mathrm{M}} \mathrm{T}_{\mathrm{R}} \mathrm{KSVM}\right)$, Technical Report, School of Industrial Engineering, University of Oklahoma, Norman, Oklahoma (2005)

15. Santosa, B., Conway, T., Trafalis, T.B.: Knowledge Based-Clustering and Application of Multi-Class SVM for Genes Expression Analysis. Intelligent Engineering Systems through Artificial Neural Networks, (2002), 12, 391 - 395

16. Hsu, C-W., Lin, C-J.: A Comparison of Methods for Multi-class Support Vector Machines. IEEE Transactions on Neural Networks, (2002) 13, 415 - 425

17. Lewis, J. M., Lakshmivarahan, S., Dhall, S.: Dynamic Data Assimilation. Cambridge University Press, (2006)

18. MATLAB User's Guide. The Math-Works, Inc., Natwick, MA 01760, (19942003). http://www.mathworks.com 